\title{
Coenurosis of Yak, Bos grunniens, caused by Taenia multiceps: A Case Report with Molecular Identification in Qinghai Tibetan Plateau Area, China
}

\author{
Xue-Yong Zhang', Ying-Na Jian†, Hong Duo, Xiu-Ying Shen, Yi-Juan Ma, Yong Fu, Zhi-Hong Guo* \\ Qinghai Academy of Animal Sciences and Veterinary Medicine, Qinghai University, State Key Laboratory of Plateau Ecology and Agriculture, \\ Qinghai University, Xining City, Qinghai Province 810016, P. R. China
}

\begin{abstract}
Coenurosis is an important zoonotic helminthic disease caused by the larval stage of the tapeworm Taenia multiceps. This parasite typically infects the brain of the intermediate hosts, including sheep, goat, cattle and even humans. We report a case of T. multiceps infection in a yak confirmed by clinical symptoms, morphological characteristics, and molecular and phylogenetic analyses. The coenurus was thin-walled, whitish, and spherical in shape with a diameter of $10 \mathrm{~cm}$. The parasite species was identified as T. multiceps by PCR amplification and sequencing of the 18S rRNA, cox 1 and nad 1 genes. Three gene sequences all showed high homology (all above 97\%) with the reference sequences from different hosts. Moreover, phylogenetic reconstructions with the 3 published Taenia gene sequences confirmed that the Qinghai yak isolate was closely related to T. multiceps. Although there are advanced diagnosis and treatment methods for coenurosis, early infection is difficult to diagnose. Importantly, the findings of yak infection case should not be ignored due to its zoonotic potential.
\end{abstract}

Key words: Taenia multiceps, coenurosis, case report, molecular identification, Qinghai Tibetan Plateau Area

\section{INTRODUCTION}

Taenia multiceps is an important zoonotic tapeworm and its larval form (Coenurus cerebralis) is the causative agent of coenurosis (Cestoda, Teniidae; Leske, 1780) [1]. The adult tapeworm inhabits the small intestine of its definitive host domestic and wild canids (dog, fox, and wolf), while the metacestode parasitizes in the brain or spinal cord of the intermediate hosts (sheep, goat, cattle, and human), and then it affects the central nervous system of the intermediate hosts [2]. Coenurosis, also known as coenuriasis, 'gid' or 'staggers', can lead to pronounced intracranial pressure, resulting in headache, ataxia, hypermetria, head deviation, blindness, stumbling, paralysis and even death of the intermediate infected sheep, goat, cattle and even humans [3-5]. Coenurosis has spread worldwide and is distributed extensively, including in Europe and the USA, and especially in developing countries in Africa and Asia,

- Received 5 March 2019, revised 11 July 2019, accepted 10 August 2019.

*Corresponding author (guozhihong65@hotmail.com)

†These authors contributed equally to this work.

(c) 2019, Korean Society for Parasitology and Tropical Medicine

This is an Open Access article distributed under the terms of the Creative Commons Attribution Non-Commercial License (http://creativecommons.org/licenses/by-nc/4.0) which permits unrestricted non-commercial use, distribution, and reproduction in any medium, provided the original work is properly cited. and results in serious socioeconomic losses to the livestock husbandry industry throughout the world [6].

In this study, we presented a rare case of yak coenurosis in the Qinghai Tibetan Plateau Area of China; emphasized the importance of animal behaviour, clinical features and molecular identification of the parasites; characterized the phylogenetic relationship of isolated parasites.

\section{CASE RECORD}

A 1.5-year-old male yak (Bos grunniens) was making irregular spinning right movements and had reduced eyesight. Upon cranial examination, the skull became soft and the skin bulged. After dissection, the skull was found to be thin, with a soft texture, and there were colorless transparent capsules with a diameter of about $10 \mathrm{~cm}$ filled with colourless, transparent water-like liquid, with white particles in the encapsulated liquid. When the capsule was removed from the brain, the affected part formed a large hollow from being under pressure for a long time (Fig. 1).

Total genomic DNA was extracted from the tissue sample using the TIANamp Genomic DNA Kit (TIANGEN Biotech, Beijing, China) according to the manufacturer's instructions. 


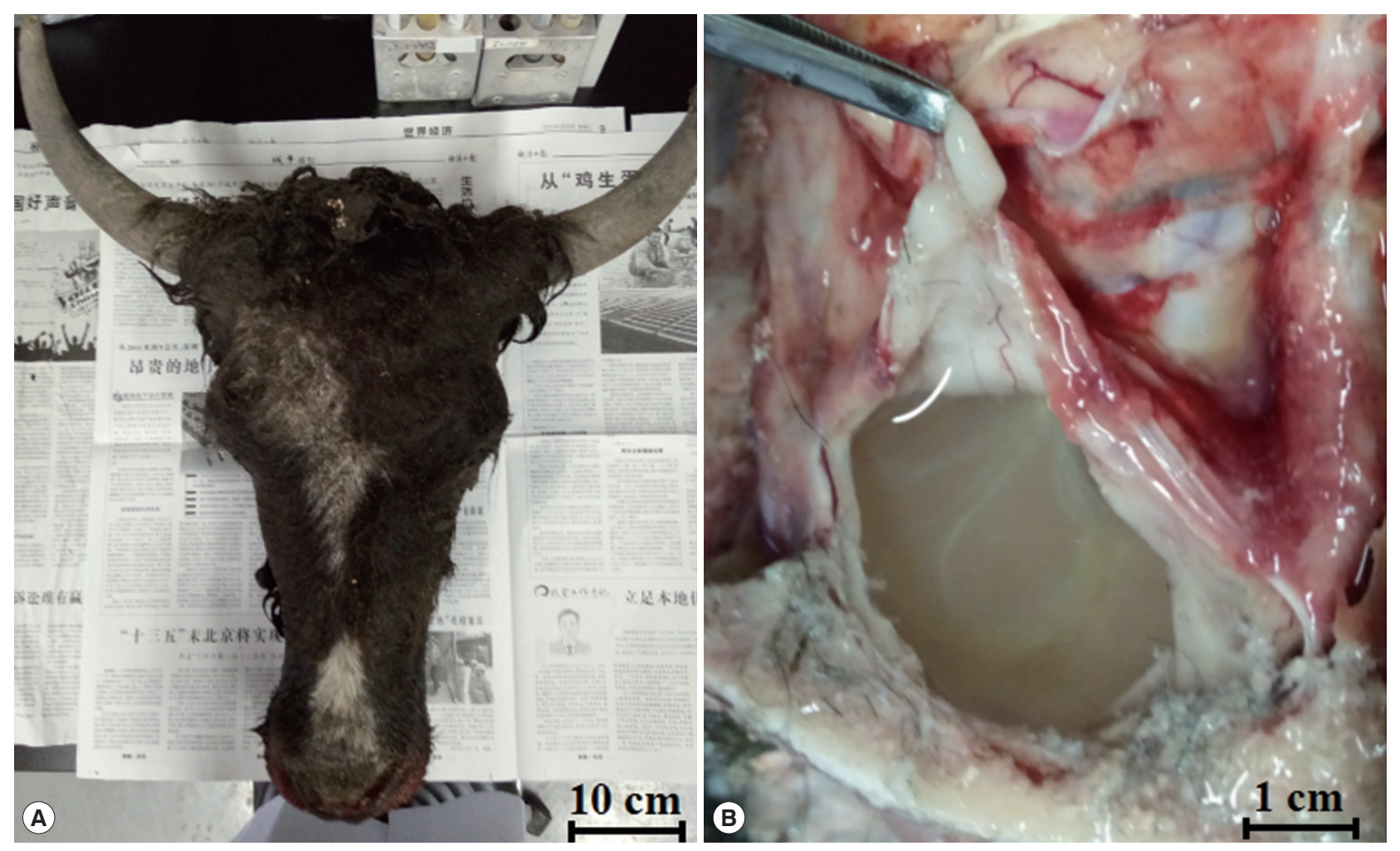

Fig. 1. The macroscopic appearance of cysts in the brain of yak ( $\mathrm{A}$ : the head of the yak; $B$ : the cyst in the brain).

PCR was performed to amplify the nuclear and mitochondrial genes with the following primers: T. multiceps $18 \mathrm{~S}$ rRNAF: $5^{\prime}$ -TCCTGCCAGTAGTCATATGC-3' and T. multiceps $18 \mathrm{~S}$ rRNAR: $5^{\prime}$ -CTTGTTACGACTTTTACTTCCTCT-3' (PCR product=2,460 bp); $T$. multiceps cox1F: $5^{\prime}$-TGTTTGAGTCATCTTATGTA- $3^{\prime}$ and T. multiceps cox1R: 5'-CAAAGGTTAATGATACAAAT-3' (PCR product=1,620 bp); T. multiceps nad1F: 5'-GGTAAATAAGTAACCAAGTC-3' and T. multiceps nad1R: 5'-AACATAAACATACCTCCC-3' (PCR product $=890$ bp). The amplification products ( $10 \mu \mathrm{l})$ were analysed by $1.5 \%$ agarose gel electrophoresis in $1 \times$ tris-acetate-EDTA buffer ( $\mathrm{pH} 8.3$ ) containing $10 \mu \mathrm{l}$ of Gelstain per $100 \mathrm{ml}$. The agarose gel was observed, and images were captured using a WD-9413B Gel Imaging Analysis System (Beijing Liuyi Biotechnology, Beijing, China).

Direct sequencing was performed by the GENEWIZ Company (Beijing, China). The obtained sequences were then aligned with reference sequences available in the GenBank database using BLAST (http://blast.ncbi.nlm.nih.gov/Blast.cgi). Phylogenetic relationships of Taenia spp. were constructed by the MEGA 5.0 software with the neighbour-joining method, performed using the Kimura 2-parameter model and 2,000 bootstrap replicates.

The species was identified as T. multiceps following the am- plification of the fragments of 3 genes, which showed very high homology to the reference sequences when blasted against the NCBI database. In detail, the 18S rRNA, cox1, and nad1 genes from the isolate were 2,467 bp, 1,623 bp, and 894 bp in length, and the $\mathrm{G}+\mathrm{C}$ contents of each gene were $55 \%$, $30 \%$, and 28\%, respectively; and the sequences of the fragments of 18S rRNA gene (MK189451), cox1 gene (MK189452) and nad1 gene (MK189454), which provided 99\% homology to $18 \mathrm{~S}$ rRNA gene of China isolate (GQ260089) and Japan isolate (AB731621), and 100\% homology to cox1 gene of China isolate (JX535576), 99\% homology to China isolate (GQ228818, FJ495086, JX507220/1/3/4/5/6/7/8/9, JX507230 /2/3/4/5/7/8/9, JX535567/8/9 and JX535570/1/2/3/5), and $99 \%$ homology to nad1 gene of China isolate (GQ228818 and KC794809/10/11). Phylogenetic reconstructions with the published 3 Taenia gene sequences showed that all T. multiceps isolates constituting a branch, and confirmed that the Qinghai yak isolate was closely related to T. multiceps based on the $18 \mathrm{~S}$ rRNA gene sequence (MK189451, Fig. 2A), cox1 gene sequence (MK189452, Fig. 2B), and nad1 gene sequence (MK189454, Fig. 2C). 
A
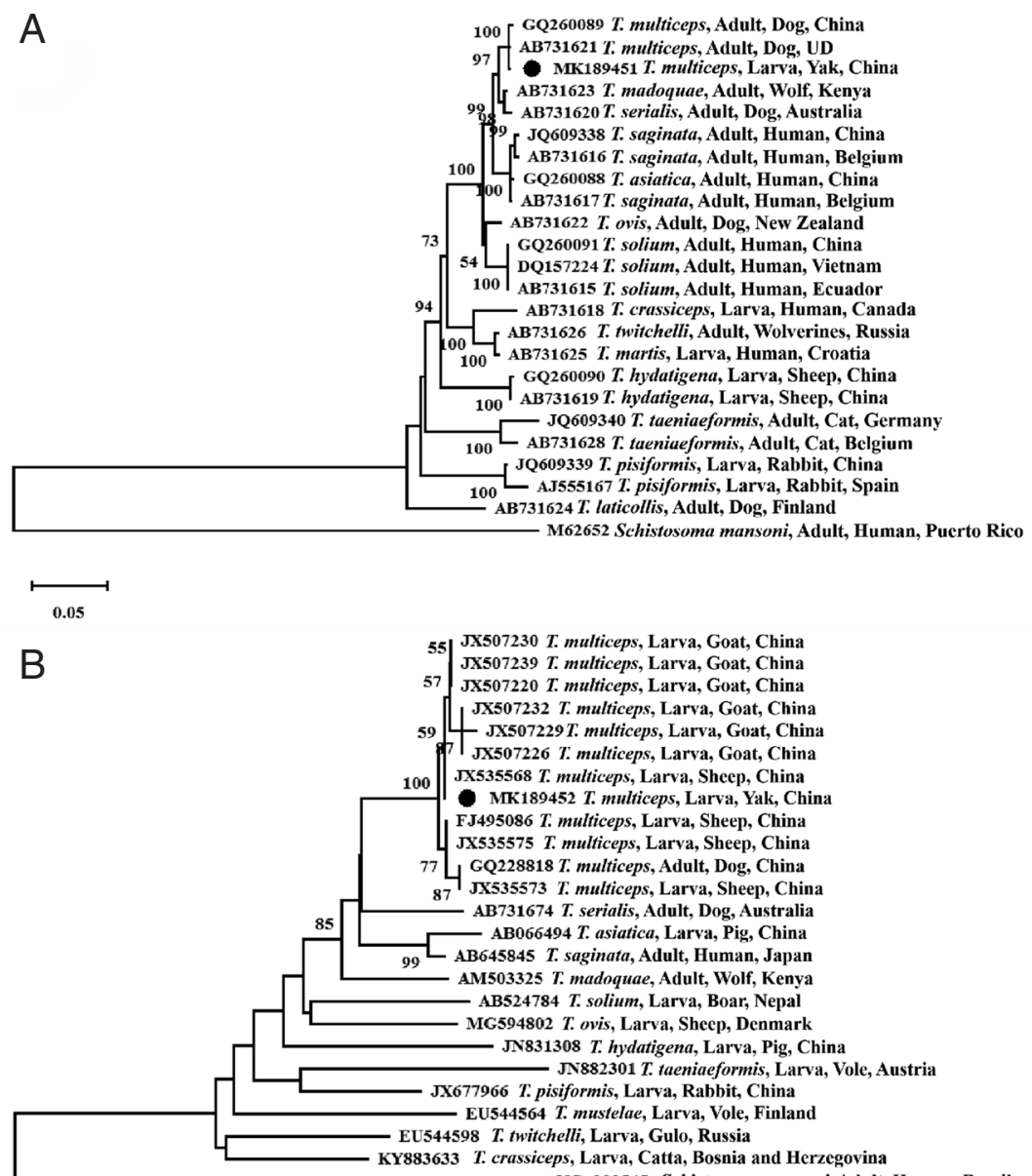
(liceps,

GQ228818 T. multiceps, Adult, Dog, China AB645845 T. saginata, Adult, Human, Japan AM503325 T. madoquae, Adult, Wolf, Kenya ABs24784 T. solium, Larva, Boar, Nepal I. ovis, Larva, Sheep, Denmark arva, Vole, Austria T. pisiformis, Larva, Rabbit, China 24598 T twitchelli, Larva, Gulo, Russia NC 002545 Schistosoma mansoni, Adult, Human, Brazil
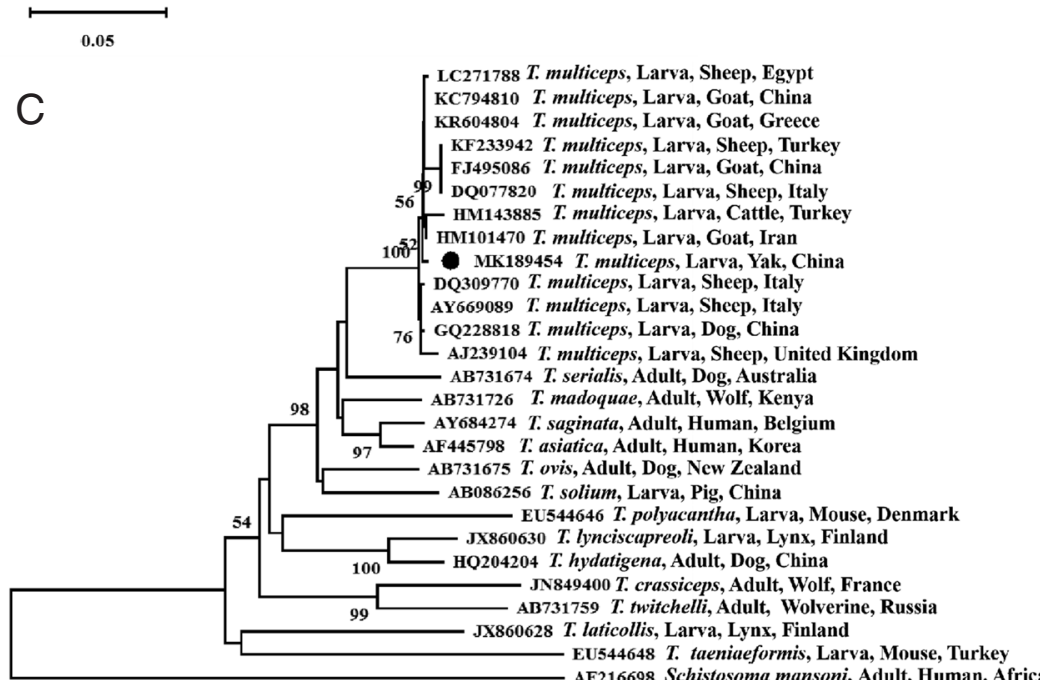

Fig. 2. Phylogenetic relationship of T. multiceps isolate obtained in the present study and other members of the genus Taenia as inferred by neighbouring-joining analysis of the 18S rRNA gene (A), cox1 gene (B) and nad1 gene (C) sequences based on genetic distances calculated by the Kimura 2-parameter model. The reliability of these trees was assessed using a bootstrap analysis with 1,000 replicates. Black circles indicate the sequences of T. multiceps from the present study. UD, Undocumented. 


\section{DISCUSSION}

Coenurosis is a group of zoonotic diseases caused by the metacestode larval stage of T. multiceps, T. serialis, and T. brauni $[3,7,8]$. The intermediate hosts, including humans and grazing animals such as cattle, sheep, goats, and rabbits, ingested the eggs in contaminated fruits, vegetables and grass, shed by the definitive hosts ( $\mathrm{dog}$, fox, and wolf).

Coenurosis is a neglected and rare disease; the recorded cases are domestic animals and human infections, and there is an existing risk of infection in humans. The yak case pathogen was identified as the larval stage of T. multiceps by clinical anatomy and molecular biology methods, and epidemiologically, there were no cases of human infections in this pastoral area. This case may be caused by wild canids (definitive hosts) because Li et al. [9] reported T. multiceps in Tibetan sand foxes in Qinghai Province of China with a 6\% prevalence; on the other hand, Feng et al. [10] isolated T. multiceps eggs from the faces of farm dogs also in Qinghai Province of China. In the same environment and area, there were many wild foxes and domestic dogs (definitive hosts) and sheep, goat and yaks (intermediate hosts) even wandered around people's residences in some situations, threatening human health.

In this case, the yak's cyst had a diameter of approximately $10 \mathrm{~cm}$, and the yak was similar to humans. When humans feel sick in the brain, they seek medical treatment and undergo brain computed tomography for diagnosis [11]. However, for the yaks, who graze freely on the grassland, when they are infected the parasite, it cannot be effectively found at the beginning stages, even when it affected the movement of the yaks, but it does not always attract the owners' attention; moreover, sometimes the owners did not realize that the yaks were infected with parasite in the brain. By the time the yaks walked in a circular motion and the symptoms of coenurosis were obvious [12], it was too late for early intervention, and the surgical treatment period was missed.

Coenurosis is a rare but extremely life-threatening disease, and coenurosis in sheep and goats were reported in China [13], but the molecular identification of T. multiceps was limited. Ten unique completed cox 1 genes of 16 T. multiceps larval isolates from goats and sheep in Gansu Province and partial cox 1 genes of 3 larval isolates from goats in Hunan Province were sequenced and showed 99\% identity [13]. The phylogenetic tree based on the $18 \mathrm{~S}$ rRNA gene, cox 1 gene and nad1 gene sequences all showed that the Qinghai yak T. multiceps isolate constituted a branch consisting the different hosts and geographical distributions showing the closest relationships, followed by other Taenia species. Therefore, the 18S rRNA gene, cox 1 gene and nad1 gene sequences can be used as molecular markers for the interspecific identification of Taenia species.

The case of yak coenurosis indicates the presence of the definitive hosts of T. multiceps in humans and domestic animals' environment, which suggests its zoonotic potential and importance to public health concerns. Considering the increasing number of domestic animals moving across provinces, even national imports and exports, coenurosis case reports could be useful for collecting a reference archive for food safety and hygiene. Therefore, more case reports and further studies on the prevalence of T. multiceps infections in sheep, goats, yaks, dogs and wildlife will still be needed in the Qinghai Tibetan Plateau Area of China for more in-depth information and insight into this disease.

\section{ACKNOWLEDGMENTS}

The authors would like to express their sincere appreciation and gratitude to the staff working at Haiyan County Animal Husbandry and Veterinary Station. This study was supported by the Research and Demonstration of Diagnosis and Prevention and Control Technology of Major Diseases of Livestock (2017NK-110), the National Natural Science Foundation of China (NO. 31860700), the Qinghai University Young and Middle Research Foundation Project (2018-QNY-1) and the Basic Scientific Independent Research Project of Qinghai Academy of Animal Science and Veterinary Medicine (MKY-2019-10).

\section{CONFLICT OF INTEREST}

The authors declare that they have no conflicts of interest concerning this work.

\section{REFERENCES}

1. Varcasia A, Tamponi C, Tosciri G, Pipia AP, Dore F, Schuster RK, Kandil OM, Manunta ML, Scala A. Is the red fox (Vulpes vulpes) a competent definitive host for Taenia multiceps? Parasit Vectors 2015; 8: 491.

2. Scala A, Varcasia A. Updates on morphobiology, epidemiology and molecular characterization of coenurosis in sheep. Parassitologia 2006; 48: 61-63.

3. Li W, Liu B, Yang Y, Ren Y, Wang S, Liu C, Zhang N, Qu Z, Yang W, 
Zhang Y, Yan H, Jiang F, Li L, Li S, Jia W, Yin H, Cai X, Liu T, McManus DP, Fan W, Fu B. The genome of tapeworm Taenia multiceps sheds light on understanding parasitic mechanism and control of coenurosis disease. DNA Res 2018; 25: 499-510.

4. Al-Riyami S, Ioannidou E, Koehler AV, Hussain MH, Al-Rawahi AH, Giadinis ND, Lafi SQ, Papadopoulos E, Jabbar A. Genetic characterisation of Taenia multiceps cysts from ruminants in Greece. Infect Genet Evol 2016; 38: 110-116.

5. Bussell KM, Kinder AE, Scott PR. Posterior paralysis in a lamb caused by a Coeneurus cerebralis cyst in the lumbar spinal cord. Vet Rec 1997; 140: 560.

6. Sharma DK, Chauhan PPS. Coenurosis status in Afro-Asian region: a review. Small Rumin Res 2006; 64: 197-202.

7. Zhang XY, Jian YN, Ma LQ, Li XP, Karanis P. A case of coenurosis in a wild rabbit (Lepus sinensis) caused by Taenia serialis metacestode in Qinghai Tibetan Plateau Area, China. Korean J Parasitol 2018; 56: 195-198.

8. Lescano AG, Zunt J. Other cestodes: sparganosis, coenurosis and Taenia crassiceps cysticercosis. Handb Clin Neurol 2013; 114: 335345.

9. Li W, Guo Z, Duo H, Fu Y, Peng M, Shen X, Tsukada H, Irie T,
Nasu T, Horii Y, Nonaka N. Survey on helminths in the small intestine of wild foxes in Qinghai, China. J Vet Med Sci 2013; 75: 1329-1333.

10. Feng K, Li W, Guo Z, Duo H, Fu Y, Shen X, Tie C, E R, Xiao C, Luo Y, Qi G, Ni M, Ma Q, Yamazaki W, Yoshida A, Horii Y, Yagi $\mathrm{K}$, Nonaka N. Development of LAMP assays for the molecular detection of taeniid infection in canine in Tibetan rural area. J Vet Med Sci 2017; 79: 1986-1993.

11. Benifla M, Barrelly R, Shelef I, El-On J, Cohen A, Cagnano E. Huge hemispheric intraparenchymal cyst caused by Taenia multiceps in a child. Case report. J Neurosurg 2007; 107: 511-514.

12. Varcasia A, Pipia AP, Arru D, Pes AM, Tamponi C, Dore F, Garippa G, Scala A. Morphological and molecular characterization of bovine coenurosis in Sardinia, Italy. Parasitol Res 2013; 112: 2079-2082.

13. Li WH, Jia WZ, Qu ZG, Xie ZZ, Luo JX, Yin H, Sun XL, Blaga R, $\mathrm{Fu}$ BQ. Molecular characterization of Taenia multiceps isolates from Gansu Province, China by sequencing of mitochondrial cytochrome c oxidase subunit 1. Korean J Parasitol 2013; 51: 197-201. 
